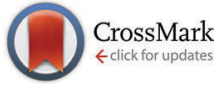

Cite this: Phys. Chem. Chem. Phys., 2014, 16, 25959

\title{
Unravelling the conformations of di-(perylene bisimide acrylate) by combining time-resolved fluorescence-anisotropy experiments and molecular modelling $\dagger$
}

\author{
F. Spreitler, ${ }^{a}$ M. Sommer, $\neq^{c}$ M. Hollfelder, ${ }^{b}$ M. Thelakkat, ${ }^{c}$ S. Gekle*b and J. Köhler*a \\ We compare the results from time-resolved fluorescence anisotropy experiments and molecular \\ modelling on perylene bisimide acrylate dimers which allows us to connect the observed spectral \\ signatures unambiguously with the non-stacked and two (parallel and anti-parallel) stacked \\ conformations. For the parallel stacked conformation the experimental data can be reproduced \\ quantitatively using a model that assumes structural relaxation in the electronically excited state of the \\ stacked aggregate. For the non-stacked conformation we find quantitative agreement between \\ experiment and modelling only if a fast hopping of the electronic excitation between the perylene \\ bisimide subunits is taken into account.
}

Received 11th July 2014

Accepted 23rd October 2014

DOI: $10.1039 / c 4 c p 03064 h$

www.rsc.org/pccp

\section{Introduction}

Exploiting the unique properties of organic semiconductors for technological applications has become one of the greatest challenges in chemical physics during the last years. ${ }^{1-4}$ Not only does the production of organic materials and devices typically need less resources and energy than their inorganic counterparts, organic semiconductors additionally offer the opportunity to open up new avenues for applications with controlled structure and tailored electronic properties. ${ }^{5} \mathrm{~A}$ prominent example is the development of new generation solar cells based on organic molecules and polymers. This task requires the design of efficient functional units for the purposes of light absorption, exciton migration, exciton dissociation, and charge collection at the electrodes. ${ }^{6-9}$ In particular, perylene bisimides (PBI) are regarded as very promising antenna dyes for the efficient harvesting of visible light. ${ }^{10-13}$ The material systems based on these molecular building blocks are attractive semiconductors with high electron affinity and strong absorption in the visible range, and the design of soluble PBIs extends from highly crystalline molecules up to non-planar amorphous

\footnotetext{
${ }^{a}$ Experimental Physics IV and BIMF, University of Bayreuth, D-95440 Bayreuth, Germany. E-mail: juergen.koehler@uni-bayreuth.de; Fax: +49-921-554002

${ }^{b}$ Physics Department, University of Bayreuth, D-95440 Bayreuth, Germany

${ }^{c}$ Applied Functional Polymers, Department of Macromolecular Chemistry I, University of Bayreuth, D-95440 Bayreuth, Germany

$\dagger$ Electronic supplementary information (ESI) available. See DOI: 10.1039/ c4cp03064h

\# Current address: Michael Sommer, Institute for Macromolecular Chemistry, Albert-Ludwigs-Universität Freiburg, 79104 Freiburg, Germany.
}

systems. ${ }^{14}$ But these important material properties have not been exploited well to obtain highly efficient devices for light harvesting or for charge separation and electron transport, for example, in solar cells for a long time. Recently, PBI dimers and trimers with specific geometry/shape have been efficiently applied in organic solar cells as acceptors instead of fullerenes. ${ }^{15-17}$ But still, the exact pathway of exciton dissipation or loss of the excitation energy of the charge-transfer states in PBI aggregates are not well studied, even though such loss channels are postulated. Information about the fundamental photophysical properties of molecular building blocks as well as their structure-function relationships are key for understanding and controlling electronic processes in organic nano-scale materials, and are accessible by optical spectroscopy. However, because the spectroscopic details depend crucially on the intermolecular interactions and the mutual orientation of the molecular building blocks better knowledge of the effects that determine the self-assembly process are required for a rational design of supramolecular functionalities. Moreover, large multichromophoric systems with great potential for applications appear very heterogeneous. Therefore many research activities have been focussed on small model systems with linear, ${ }^{18-20}$ cofacial, ${ }^{21}$ zigzag, ${ }^{22-25}$ cyclic, ${ }^{26}$ or square geometries ${ }^{27}$ as well as dendrimers consisting of 2 or more subunits. ${ }^{28,29}$

Recently, we have studied dyads consisting of PBI antenna molecules that were covalently linked to a fullerene by a flexible hexyl spacer and found an efficient energy transfer from the antennae to the fullerene, while charge-transfer processes play only a minor role. ${ }^{30}$ In addition, we observed triplet-triplet energy transfer back from the fullerene to the perylene bisimide. However, a significant improvement of the light-harvesting 
efficiency by simply increasing the number of antenna units per $\mathrm{C}_{60}$ is prohibited due to a strong face-to-face $\pi-\pi$-stacking of the PBIs which drastically alters their photophysical properties, ${ }^{31}$ and which might have tremendous impact on the performance of actual devices built from these materials. On the other hand, the flexibility/rigidity of the chemical structural units influence the domain sizes of structures formed in thin films. This is of particular importance if flexible materials, such as polymers, are used to construct solar cells, where the domain size determines the interface and charge transport pathways required for charge separation and transport, respectively. In order to study the influence of aggregation on the photophysical properties of such constructs, we have investigated flexible dimers of PBI acrylates, (PerAcr) $)_{2}$, dissolved in toluene by time-resolved spectroscopy and fluorescencecorrelation spectroscopy (FCS). ${ }^{32}$ The time-resolved data allowed unambiguously to distinguish between an aggregated and a non-aggregated conformation of (PerAcr) $)_{2}$, referred to hereafter as AGG-(PerAcr $)_{2}$ and ISO-(PerAcr $)_{2}$, respectively. For the nonaggregated conformation, ISO-(PerAcr) $)_{2}$, both the fluorescenceexcitation and the emission spectrum (obtained as a decay associated spectrum (DAS) from time-resolved data) closely resembled those of the monomeric PerAcr. In contrast, the aggregated conformation, AGG-(PerAcr $)_{2}$, featured a modified fluorescence-excitation spectrum and an emission that was strongly red shifted with respect to the emission from ISO$(\text { PerAcr })_{2}$, see Fig. 1. This strong red-shift was exploited experimentally to distinguish between the emission from the ISO and AGG conformations through selective detection of fluorescence with wavelengths between 510 and $550 \mathrm{~nm}$ (green spectral range) and above $660 \mathrm{~nm}$ (red spectral range). Similarly, the fluorescence lifetime of ISO-(PerAcr $)_{2}$ amounted to 3.7 ns close to the 4 ns observed for PerAcr, whereas the lifetime of AGG-(PerAcr) $)_{2}$

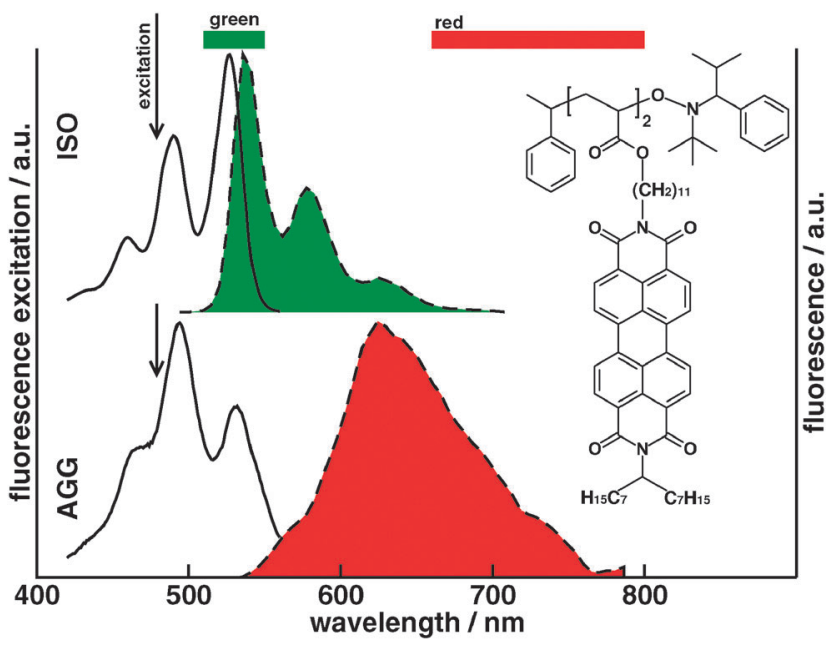

Fig. 1 Fluorescence-excitation spectra (full lines) and decay associated emission spectra (dashed lines) of ISO-(PerAcr) 2 (top) and AGG-(PerAcr) 2 (bottom). For better visibility all spectra have been peak normalised. The decay-associated spectra (DAS) were obtained for an excitation wavelength of $479 \mathrm{~nm}$ as denoted by the arrows. The bars on top of the figure refer to the two spectral bands that were used for discriminating green and red emission, and the inset shows a sketch of the (PerAcr $)_{2}$ structure. was 27 ns. However, the FCS data could only be explained consistently if we predicted a further (PerAcr) $)_{2}$ species, referred to as NF-(PerAcr $)_{2}$, whose fluorescence was quenched faster than the experimental time resolution of 30 ps. From a couple of control experiments we were able to exclude NF-(PerAcr $)_{2}$ being a charge transfer or a triplet state.

Here we are aiming at a better understanding of the structureproperty relationships of the various conformations and to shed more light on the character of the NF state. In order to do so we performed time-resolved fluorescence-anisotropy experiments on (PerAcr $)_{2}$ in combination with molecular modelling. This approach allows us to interpret the experimental results based on the structures of the aggregated conformations of (PerAcr $)_{2}$ consistently. Interestingly, the non-fluorescent state can be assigned to a meta-stable (sub-) conformation of the (PerAcr) $)_{2}$, where the two PBI units are stacked in an anti-parallel orientation with respect to each other. Moreover, we come to the conclusion that the fast depolarisation observed for the signal from the nonaggregated conformation ISO-(PerAcr) ${ }_{2}$ reflects a fast hopping of the excitation energy between the two non-stacked PBI chromophores. The current study illustrates that subtle differences in the aggregation of the two PBI units (parallel/anti-parallel) result in drastic consequences for the photophysical properties of the entire aggregate.

\section{Methods}

\section{Sample preparation}

PerAcr and (PerAcr $)_{2}$ were synthesised as described earlier. ${ }^{32}$ The fluorescence anisotropy experiments were performed on solutions of PerAcr or (PerAcr $)_{2}$ in toluene at concentrations of $1 \mu \mathrm{M}$.

\section{Optical setup}

The time-resolved fluorescence experiments were conducted on a modified version of the streak-camera setup described earlier. $^{32}$ The samples were excited at $479 \mathrm{~nm}$ with the frequency-doubled output from a titanium:sapphire laser system (Tsunami, Newport Spectra-Physics) that provided pulses of 2 ps duration. Frequency doubling as well as controlling of the repetition rate of the laser between $200 \mathrm{kHz}$ and $2 \mathrm{MHz}$ was accomplished using a pulse picker (Model 3980, Newport Spectra-Physics). The excitation energy per pulse was adjusted not to exceed $10 \mathrm{pJ}$. The polarisation of the excitation light was adjusted vertically with respect to the laboratory coordinate system (i.e. perpendicular to the optical table) with a half-wave plate. Two achromatic lenses were used to focus the excitation onto the sample and to collect the emission under right-angle conditions. In order to measure the parallel and the perpendicular component of the fluorescence from the sample simultaneously we used two broadband polarising beam splitters that were slightly tilted with respect to each other. This separated the emission from the sample into two mutually orthogonally polarised beams that propagated under a small angle towards the entrance slit of a streak camera system 
(C5680 series, Hamamatsu) allowing for simultaneous detection of both polarization components next to each other on the streak camera. The streak system was operated in single sweep mode and provided a temporal resolution of $30 \mathrm{ps}$, while the vertical axis of the streak image covered the time interval until $4.5 \mathrm{~ns}$ after the excitation pulse. Spectral selectivity in the detection path was achieved by using dielectric filters (green spectral region (510-550 nm), Brightline HC 531/40, AHF; red spectral region (660 $\mathrm{nm}$ and above), EdgeBasic $635 \mathrm{LP}, \mathrm{AHF}$ ). The analysis of the data was performed using the freely distributed software gnuplot (version 4.2.3).

\section{MD simulation}

The simulated system consisted of 1000 toluene molecules solvating a single (PerAcr) $)_{2}$ dimer. Simulations were run using GROMACS 4.5 and $4.6^{33}$ with temperature and pressure fixed at $300 \mathrm{~K}$ and $1013 \mathrm{hPa}$, respectively, by a Berendsen algorithm. Molecular topologies were obtained from the Automated Topology Builder ${ }^{34}$ for the PBI dimer depicted in Fig. 1. Electrostatic interactions were calculated using Particle Mesh Ewald summation. For short-ranged Lennard-Jones interactions a shifted cut-off at $1 \mathrm{~nm}$ was chosen. The time step was 2 fs. The anisotropy decay shown in Fig. 5 was obtained from 34 runs over a total time of $2700 \mathrm{~ns}$ that the systems spent in the ISO state.

The free energy profile in Fig. 3f has been calculated using the Umbrella Sampling capability of GROMACS. The distance between the centers-of-mass of the two monomers is restricted by strong harmonic springs around the stacked value of $0.375 \mathrm{~nm}$. The profile has been calculated using 14 windows and applying a harmonic umbrella potential for the angle within each window.

In order to match the time scales between MD and experiment, all times extracted from MD simulations were divided by a constant factor 2.5 representing the simulation/experiment ratio for the long-time anisotropy decay of a single PerAcr monomer. By using the anisotropy decay of a single PerAcr monomer as a reference time scale we were able to work out unambiguously the differences between PerAcr and (PerAcr) $)_{2}$ which are the main focus of this work. For more details see the ESI. $\dagger$

\section{Results}

\section{Non-exponential associated anisotropy decay of (PerAcr $)_{2}$}

In Fig. 2 we show the decay of the fluorescence of (PerAcr) $)_{2}$ as a function of the polarisation of the emission for the green (Fig. 2a) and the red (Fig. 2b) spectral detection channel. Thereby parallel $(\|)$ and perpendicular $(\perp)$ refer to the polarisation of the emission with respect to the polarisation direction of the excitation light. For short times after the excitation pulse the anisotropic character of the emission is apparent. The shape of the anisotropic part of these fluorescence decays can be studied in more detail in Fig. 2c which shows the first 200 ps after excitation, as indicated by the boxed regions in Fig. $2 a$ and $b$, on an expanded scale, together with the predictions from two models that will be discussed later.

From these data we have calculated the time-resolved fluorescence anisotropy $r(t)$ according to ${ }^{35}$

$$
r(t)=\frac{I_{\|}(t)-I_{\perp}(t)}{I_{\|}(t)+2 \cdot I_{\perp}(t)}
$$

as shown in the insets of Fig. $2 \mathrm{a}$ and $\mathrm{b}$ on a semi-logarithmic scale. For the emission in the green spectral region, the anisotropy decays non-exponentially from an initial value of 0.3 and approaches asymptotically an exponential decay with a time constant of 700 ps after about 500 ps. Similarly, in the red detection channel the anisotropy decays non-exponentially, yet with an initial value of 0.2 , and approaches asymptotically the same exponential as for the green detection channel.

As a reference, we repeated the experiment on the monomeric PerAcr (data not shown). The PerAcr anisotropy decays non-exponentially from an initial value of nearly 0.4 , and approaches an exponential decay with a time constant of 500 ps after about 200 ps. This behaviour did not depend on the wavelength of the detected light.

\section{Geometry of ISO, AGG and NF conformations from MD simulation}

In the following we will relate the states ISO, AGG and NF observed previously ${ }^{32}$ to the different geometric conformations of the molecule depicted in Fig. 3a-c. As starting configuration for the Molecular Dynamics (MD) simulations for the PBI dimer (PerAcr $)_{2}$ we choose arbitrary non-stacked states such as the one depicted in Fig. 3a. Here, the two monomers are widely separated corresponding to the experimental ISO state described above. Fig. 3d shows a typical time trace of the vertical distance between the two monomers, which at first fluctuates wildly. After about $22 \mathrm{~ns}$ a transition to an aggregated state occurs for which the vertical distance locks to a fixed average value of $0.36 \mathrm{~nm}$. This vertical distance corresponds closely to the $\pi-\pi$ stacking distance of PBI monomers found in classical $^{36,37}$ and $a b$ initio $^{38} \mathrm{MD}$ simulations as well as X-ray diffraction experiments. ${ }^{39}$

Next we consider the orientation of the two monomer units with respect to each other by inspecting the vectors connecting the two nitrogen atoms within each monomer referred to as $\mathrm{NN}$-vector in the following. Fig. 3e shows the time trace of the angle $\alpha$ between these two NN-vectors. This additional geometric quantity reveals that the aggregated state contains in fact two sub-conformations. In the first sub-conformation the angle fluctuates around an average value of $37^{\circ}$ corresponding to an arrangement in which the alkane chains connecting the two monomers are attached at the same end of each monomer and the two NN-vectors are thus oriented parallel (Fig. 3b). In the second sub-conformation, the average value is approximately $135^{\circ}$ such that the attachment points of the alkane chains lie on opposite sides of the two monomers and the two NN-vectors are oriented anti-parallel (Fig. 3c). The occurrence of the anti-parallel sub-conformation is unique to the PBI dimer due to the symmetry breaking by the attached 
a)

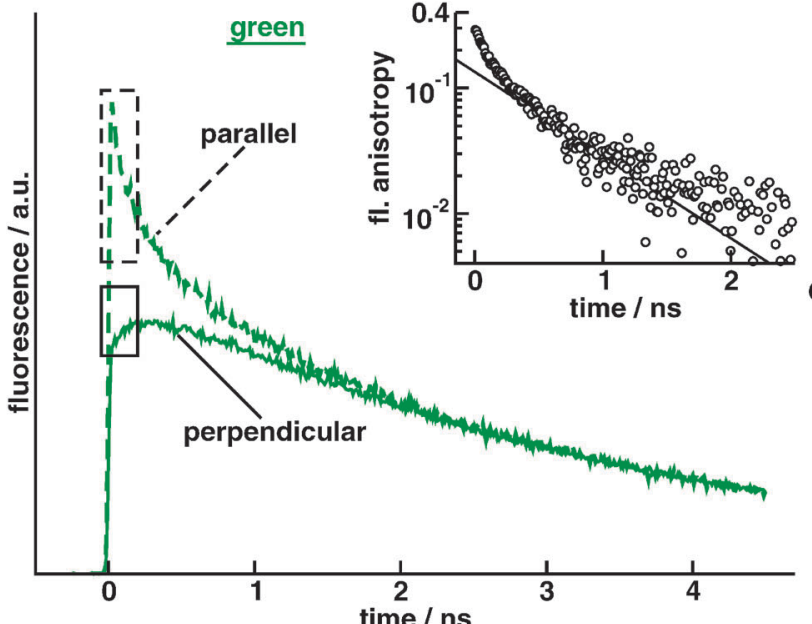

b)

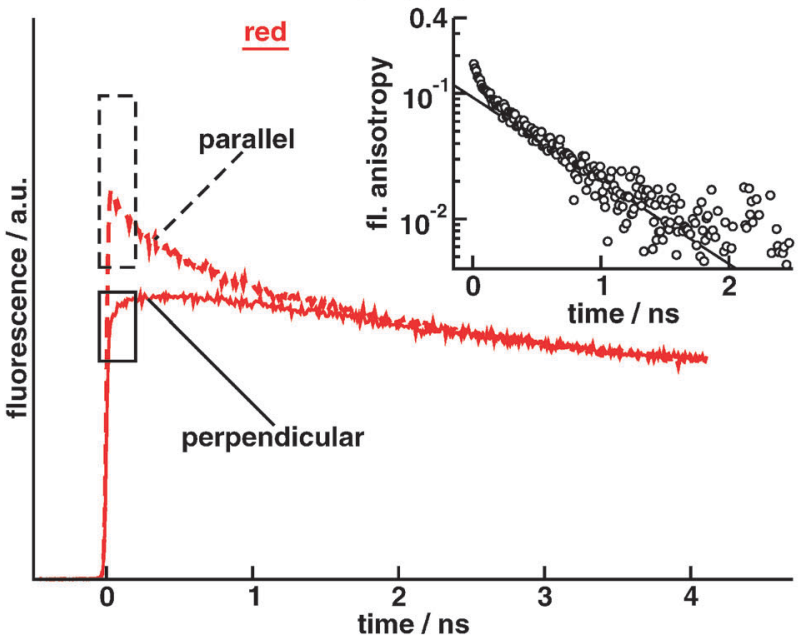

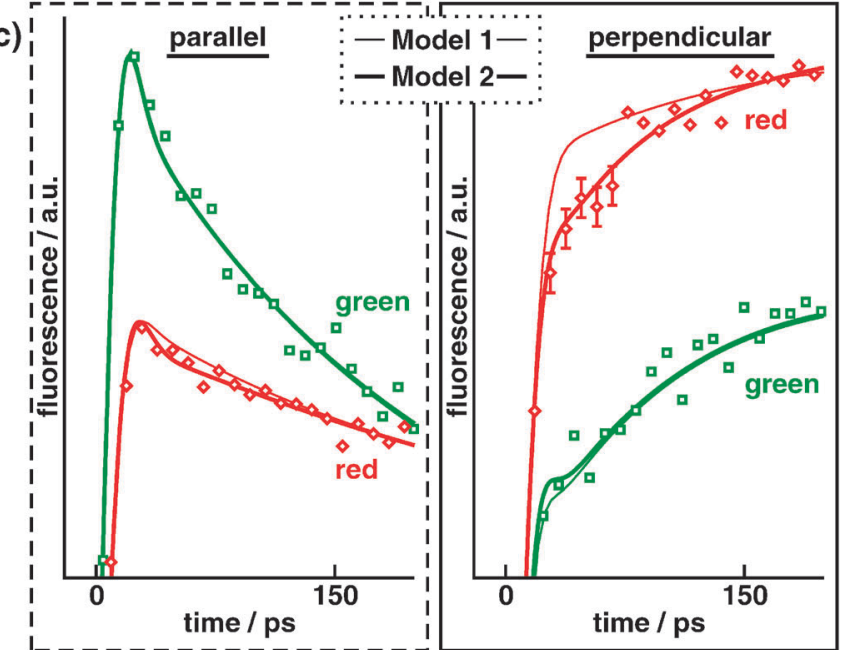

Fig. 2 Polarisation-resolved fluorescence decay of (PerAcr $)_{2}$. The emission is detected (a) in the green spectral range $(510-550 \mathrm{~nm})$ and $(\mathrm{b})$ in the red spectral range (above $660 \mathrm{~nm}$ ). All decays have been normalised such that their tails overlap. The insets in (a) and (b) show the fluorescence anisotropy decay that has been calculated from the polarisation-resolved decays according to eqn (1). The solid line corresponds to a mono-exponential decay with a decay time of 700 ps as a guide for the eye. (c) Enlarged view of the boxed regions in (a) and (b) for the parallel (left) and perpendicular (right) polarised emission in the respective spectral ranges as indicated by the colour code. The diamonds and squares refer to the experimental data, and the lines refer to two models discussed in the text. For those data points that deviate significantly from the predictions of model 1 (thin line), the error bars are provided.

alkane chains and does not exist for a stack of symmetric PerAcr monomers. We note that the anti-parallel conformation is not observed in every simulation, as it is rather common that a system starting in the open configuration directly folds into the parallel aggregated conformation without passing through the anti-parallel conformation. We attribute the less stable character of the anti-parallel sub-conformation to the entropically unfavourable stretching of the alkane chain which counteracts the (favourable) $\pi-\pi$ stacking energy. This is further corroborated by Fig. $3 \mathrm{f}$ where we show the free energy profile as a function of the angle $\alpha$ while keeping the distance between the two monomers fixed. The two minima corresponding to the parallel and anti-parallel states, respectively, are clearly visible. While the angle at which the parallel minimum is observed matches exactly with the average angle of the parallel configuration $\left(37^{\circ}\right)$ from Fig. 3e, the angular position of the antiparallel minimum lies between $140^{\circ}$ and $150^{\circ}$ and is thus slightly larger than the average value in the anti-parallel configuration of $135^{\circ}$ from Fig. 3e. We attribute this to the larger distance fluctuations in the anti-state ( $c f$. Fig. 3d) which are not allowed during the calculation of the free energy profile as described in the Methods section. The parallel configuration exhibits a significantly lower free energy which explains its more stable character.

We now proceed to connect the three conformations in Fig. 3 to the three conformations observed in earlier experiments. ${ }^{32}$ For obvious reasons, the open and the parallel aggregated states (Fig. 3a and b) can be identified with the ISO and AGG states, respectively. We tentatively identify the anti-parallel conformation in Fig. 3c with the NF state observed in the experiments. The reason for doing so becomes clear when we consider the standard deviation of the angle $\alpha$ from its average value of $135^{\circ}\left(37^{\circ}\right)$ in the anti-parallel (parallel) conformations. In the anti-parallel case we find a standard deviation of $20^{\circ}$ 
a) open (ISO)

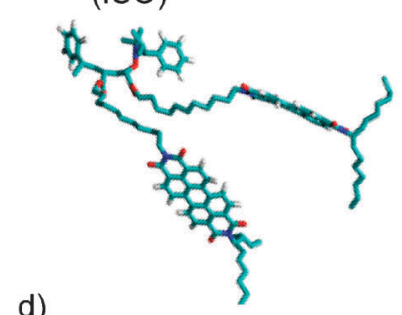

d)

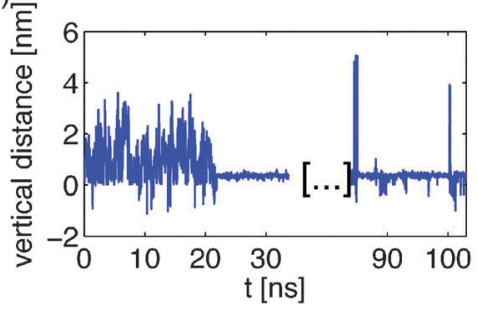

b)

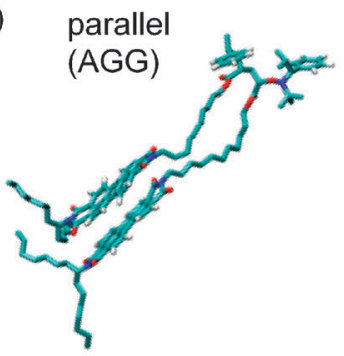

e)

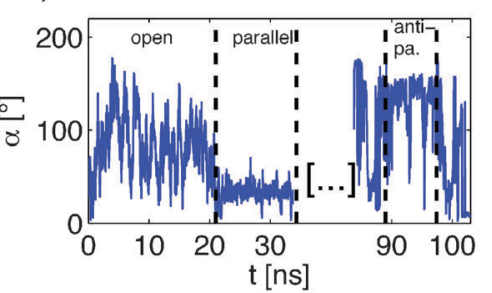

c) anti-parallel

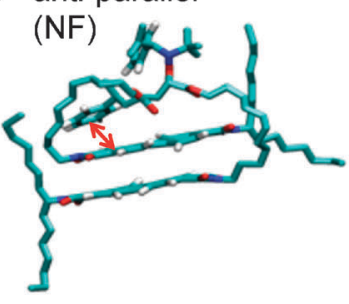

f)

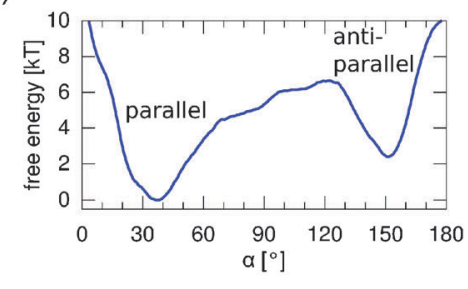

Fig. 3 (a)-(c) Snapshots of the three configurations observed in MD simulations. The double-headed arrow in (c) indicates the distance between the aromatic end group and one of the PBI chromophores. (d) The vertical distance between the two monomers allows us to distinguish between open and aggregated conformations. (e) The time trace of the angle between the NN connection vectors then reveals the existence of the two aggregated sub-conformations with mutually parallel and anti-parallel NN-vectors. (f) The free energy as a function of the rotation angle clearly shows the existence of the two states and the more stable character of the parallel orientation.

which is almost twice as large as in the parallel conformation where we find only $12^{\circ}$. In a similar fashion, the vertical distance in the anti-parallel conformation fluctuates more strongly as can be seen in Fig. 3d. These stronger fluctuations together with a possible interaction between the benzene rings at the ends of the polymer chain and the PBI molecules (marked by the red arrow in Fig. 3c) may lead to quenching of the fluorescence in the anti-parallel conformation. ${ }^{40,41}$

\section{Data analysis}

The decay of the anisotropy differs for the two spectral detection channels, see Fig. 2, which indicates that the dynamics of the two fluorescent conformations that emit predominantly in either spectral region differs significantly. While a clear correspondence between the emission in the green spectral region and the ISO conformation could be established, such a correspondence between the AGG conformation and the emission in the red spectral region does not exist due to non-negligible crosstalk. Previously, we found that the time-integrated contribution of the emission from the ISO conformation into the red detection channel was about $4 \%$ of the total intensity, which corresponds to an amplitude of $30 \%$ for this component in the multi-exponential fluorescence decay. As a consequence of this, we here consider contributions from all conformations $\mathrm{K}$ (ISO, AGG, or NF) into both spectral detection channels $\mathrm{n}$ (green or red) to the emitted fluorescence intensity. Hence, from the definition of the fluorescence anisotropy, eqn (1), we deduce

$$
\begin{aligned}
& I_{\|}^{(n)}(t) \propto \sum_{K} a_{K}^{(n)} \cdot f_{K}(t) \cdot\left(1+2 \cdot r_{K}(t)\right) \\
& I_{\perp}^{(n)}(t) \propto \sum_{K} a_{K}^{(n)} \cdot f_{K}(t) \cdot\left(1-r_{K}(t)\right)
\end{aligned}
$$

for the fluorescence intensity of the respective polarisation in the spectral region n. Here $a_{K}^{(n)}$ denotes the relative amplitude of conformation $K$ in the detection channel $n, \sum_{K} a_{K}^{(n)}=1, f_{K}(t)$ is the fluorescence decay of conformation $K, r_{K}(t)$ is the fluorescence anisotropy decay of conformation $K$, and the sum runs over all three conformations. We imply that all changes between the conformations $K$ take place in the electronic ground state of the dimers. This is justified because the time scale for the conformational changes is at least 5 times longer as the longest decay time found for the electronically excited states. ${ }^{32}$ Hence, we used the following parametrisation to model the measured polarisation-resolved decay curves of $(\text { PerAcr })_{2}$ presented in Fig. 2,

$$
I_{p}^{(n)}(t)=A_{p}^{(n)} \cdot\left(\sum_{K} a_{K}^{(n)} \cdot f_{K}(t) \cdot\left(1+z_{p} \cdot r_{K}(t)\right)\right) \otimes \operatorname{IRF}_{p}^{(n)}(t) .
$$

Here, $p$ refers to the polarisation of the emission (either $\|$ or $\perp$ ), the term between the parenthesis corresponds to eqn (2) using $z_{\|}=2$, and $z_{\perp}=-1, A_{p}^{(n)}$ are the amplitudes of the decays, $\otimes$ denotes a convolution, and $\operatorname{IRF}_{p}^{(n)}(t)$ represents the instrument response function which is modelled as a Gaussian. In order to minimize the number of free parameters the fitting was conducted as a global fit where we determined simultaneously a single set of fit parameters for all four fluorescence decay curves. Since it has been found that the decay of the NF conformation is much faster than the temporal resolution of the experiment, this fluorescence decay is always modelled as a delta function. In the following we will present two models that have been tested to explain the data. 
The simplest model to start with is to assume that upon optical excitation of any conformation all relaxation processes are faster than the experimental time resolution and that the subsequent fluorescence decays from the ISO and AGG conformations are single exponentials. Hence, we use

$$
\begin{aligned}
f_{\mathrm{AGG}}(t) & =\mathrm{e}^{-\frac{t}{\tau_{\mathrm{AGG}}}} \\
f_{\mathrm{ISO}}(t) & =\mathrm{e}^{-\frac{t}{\tau_{\mathrm{ISO}}}} \\
f_{\mathrm{NF}}(t) & =\delta(t)
\end{aligned}
$$

with, $\tau_{\mathrm{AGG}}=27 \mathrm{~ns}$ and $\tau_{\mathrm{ISO}}=3.7 \mathrm{~ns}$, as determined earlier. ${ }^{32}$ The corresponding decays of the anisotropy are modelled as sums of exponentials. Yet, it turns out that only for the ISO conformation a bi-exponential decay of the anisotropy is needed to properly describe the data, and that the decay of the anisotropy of the AGG conformation can be treated as a single exponential. This reflects that the observed non-monoexponential decay of the anisotropy in the red detection channel, see inset Fig. 2b, stems solely from crosstalk of the ISO conformation. Since the NF conformation decays faster than the temporal resolution, only its initial anisotropy directly after excitation is detectable and we treat the NF anisotropy as a constant. Hence

$$
\begin{aligned}
r_{\mathrm{AGG}}(t) & =r_{\mathrm{AGG}} \cdot \mathrm{e}^{-\frac{t}{\theta_{\mathrm{AGG}}}} \\
r_{\mathrm{ISO}}(t) & =r_{\mathrm{ISO}, 1} \cdot \mathrm{e}^{-\frac{t}{\theta_{\mathrm{ISO}, 1}}}+r_{\mathrm{ISO}, 2} \cdot \mathrm{e}^{-\frac{t}{\theta_{\mathrm{ISO}, 2}}} \\
r_{\mathrm{NF}}(t) & =r_{\mathrm{NF}}
\end{aligned}
$$

Here $r_{\mathrm{AGG}}, r_{\mathrm{ISO}, 1}, r_{\mathrm{ISO}, 2}$, and $r_{\mathrm{NF}}$ are the initial anisotropies of the three conformations AGG, ISO, and NF respectively, and $\theta_{\mathrm{AGG}}$, $\theta_{\text {ISO }, 1}, \theta_{\text {ISO, } 2}$ are the corresponding correlation times. The experimental observation that the initial amplitudes of the experimental anisotropy decays deviate from the fundamental anisotropy of 0.4 , is taken into account by treating $r_{\mathrm{AGG}}, r_{\mathrm{ISO}, 1}, r_{\mathrm{ISO}, 2}$ and $r_{\mathrm{NF}}$ as free parameters. However, it turned out that the results of the fits did not depend significantly on the value of $r_{\mathrm{NF}}$ preventing an unambiguous determination of this parameter. Therefore we used here arbitrarily $r_{\mathrm{NF}}=0.4$. The result of the fit is shown in Fig. $2 \mathrm{c}$ by the thin line. This simple model, referred to as model 1 in the following, already reproduces the observations with reasonable accuracy. However, for the perpendicularly polarised emission in the red spectral region a small yet significant deviation of the data points from the model can be observed during the first $100 \mathrm{ps}$ after the excitation pulse, see Fig. 2c.

In order to further improve the modelling of our data we take advantage of work that was conducted on stacks of PBI molecules. $^{38,42,43}$ Combining ultrafast laser spectroscopy with newly developed quantum-chemistry calculations it was revealed that the electronic excitations of a stack of PBI molecules correspond to delocalised exciton states that become localised within femto-seconds on two PBI subunits. Interestingly, the subsequent electronic relaxation is accompanied by a reorganisation of the two PBI subunits. Initially the long axes of

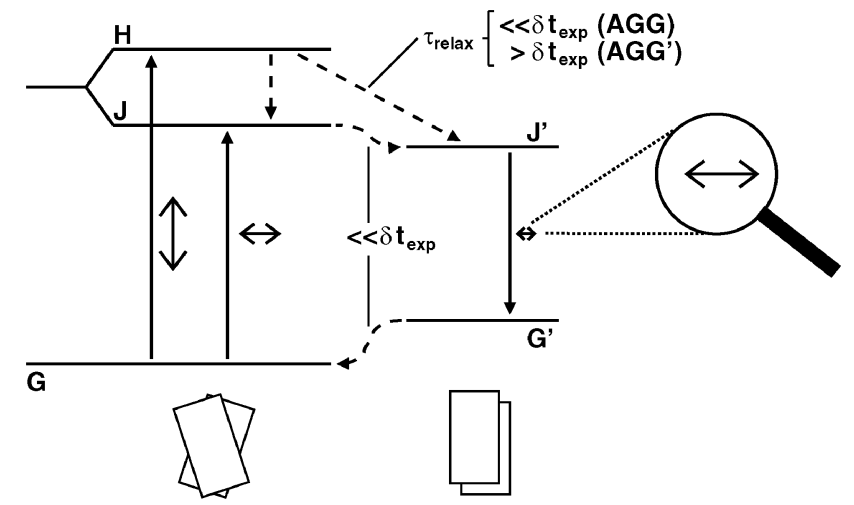

Fig. 4 Energy level diagram of the aggregated conformation of (PerAcr) $)_{2}$. $\mathrm{G}, \mathrm{J}$, and $\mathrm{H}$ refer to the ground state and the two lowest electronically excited states for the tilted conformation of the two monomers, respectively. $J^{\prime}$ and $G^{\prime}$ refer to the lowest electronically excited state and the ground state after the molecular relaxation of the monomers into a parallel configuration. The full arrows refer to radiative transitions between these states, whose relative strengths and orientations of the transition-dipole moments are indicated by the double-headed arrows. The wavy dashed arrows between $\mathrm{J}^{\prime}$ and $\mathrm{J}$ and $\mathrm{G}^{\prime}$ and $\mathrm{G}$ refer to the molecular relaxation that occurs on time scales faster than the experimental time resolution $\delta t_{\text {exp }}$. Relaxation of the electronically excited state $\mathrm{H}$ can occur to $\mathrm{J}$ and $\mathrm{J}^{\prime}$. For model 1 the assumption is that none of these processes can be resolved experimentally, whereas for model 2 it is taken into account that for a part of the population of $H$ (denoted as $A G G^{\prime}$ ) the relaxation from $\mathrm{H} \rightarrow \mathrm{J}^{\prime}$ occurs with a time constant $\tau_{\text {Relax }}$ that can be resolved experimentally. For more details see text.

the two stacked PBI monomers are oriented under an angle of about $30^{\circ}$ with respect to each other, which is in close agreement with the value of $37^{\circ}$ that we find in the MD simulations for the AGG conformation, see Fig. 3. Few picoseconds after the optical excitation and electronic relaxation, the two PBI subunits reorient into a configuration where the long axes of the molecules are oriented parallel with respect to each other. Based on these results we use the energy level diagram for the aggregated dimer that is shown in Fig. 4 to motivate a modification of the model presented above.

In the electronic ground state $\mathrm{G}$ of the aggregated conformation the two monomers are tilted with respect to each other and the lowest delocalised exciton states of the aggregated conformation are denoted as $\mathrm{J}$ and $\mathrm{H}$, respectively, according to the mutual orientation of the transition-dipole moments of the monomers. We note that the transitions $\mathrm{H} \leftarrow \mathrm{G}$ and $\mathrm{J} \leftarrow \mathrm{G}$ can be excited with light whose linear polarisations are mutually orthogonal. Upon excitation of the aggregated conformation, either into the $\mathrm{J}$ or the $\mathrm{H}$ state, the system relaxes to $\mathrm{J}^{\prime}$, i.e. the lowest electronically excited state after the molecular relaxation where the two PBI monomers are oriented parallel with respect to each other. The radiative decay of this state $\left(\mathrm{J}^{\prime} \rightarrow \mathrm{G}^{\prime}\right)$ occurs on a relatively slow timescale due to the small transition-dipole moment that is associated with this transition in this configuration. According to ref. 42, there are two pathways from $\mathrm{H}$ to $\mathrm{J}^{\prime}$ (i) the charge-transfer mediated transition to the $\mathrm{J}$ state on a time-scale of $200 \mathrm{fs}$ which is followed by torsional relaxation to $\mathrm{J}^{\prime}$, and (ii) a direct "self-trapping" transition $\mathrm{H} \rightarrow \mathrm{J}^{\prime}$ for which 
no timescale is given. In contrast to model 1 , where it was implicitly assumed that the relaxation between absorption and emission of the aggregated dimers was instantaneous, we now introduce an additional component in the fluorescence decay of the aggregated dimers, $\mathrm{AGG}^{\prime}$, for which the relaxation from state $\mathrm{H}$ to state $\mathrm{J}^{\prime}$ occurs on a timescale that can be experimentally resolved, and where $\tau_{\text {relax }}$ denotes the time constant for this process. For brevity the modified model will be termed model 2 hereafter. Accordingly, the fluorescence and anisotropy decays are modelled as

$$
\begin{gathered}
f_{\mathrm{AGG}}(t)=\mathrm{e}^{-\frac{t}{\tau_{\mathrm{AGG}}}} \\
f_{\mathrm{AGG}^{\prime}}(t)=\mathrm{e}^{-\frac{t}{\tau_{\mathrm{AGG}}}}-\mathrm{e}^{-\frac{t}{\tau_{\mathrm{relax}}}} \\
f_{\mathrm{ISO}}(t)=\mathrm{e}^{-\frac{t}{\tau_{\mathrm{ISO}}}} \\
f_{\mathrm{NF}}(t)=\delta(t) \\
r_{\mathrm{AGG}}(t)=r_{\mathrm{AGG}} \cdot \mathrm{e}^{-\frac{t}{\theta_{\mathrm{AGG}}}} \\
r_{\mathrm{AGG}}(t)=r_{\mathrm{AGG}} \cdot \mathrm{e}^{-\frac{t}{\theta_{\mathrm{AGG}}}} \\
r_{\mathrm{ISO}}(t)=r_{\mathrm{ISO}, 1} \cdot \mathrm{e}^{-\frac{t}{\theta_{\mathrm{ISO}, 1}}}+r_{\mathrm{ISO}, 2} \cdot \mathrm{e}^{-\frac{t}{\theta_{\mathrm{ISO}, 2}}} \\
r_{\mathrm{NF}}(t)=r_{\mathrm{NF}}
\end{gathered}
$$

For this approach $K$ in eqn (3) runs over ISO, AGG, AGG' and $\mathrm{NF}$, where AGG refers to the fraction of aggregated dimers for which the $\mathrm{H} \rightarrow \mathrm{J}^{\prime}$ relaxation cannot be resolved experimentally, and $\mathrm{AGG}^{\prime}$ to the fraction of aggregated dimers that decays with the time constant $\tau_{\text {Relax }}$ to $\mathrm{J}^{\prime}$. Moreover, $r_{\mathrm{AGG}^{\prime}}$ represents the initial anisotropy of $\mathrm{AGG}^{\prime}$. At this stage we cannot specify whether the fractions $\mathrm{AGG}$ and $\mathrm{AGG}^{\prime}$ correspond to two different conformations of aggregated (PerAcr) $)_{2}$, or whether they simply represent the two different decay channels for the aggregated conformation from the $\mathrm{H}$ towards the $\mathrm{J}^{\prime}$ state. The idea is that after the relaxation to $\mathrm{J}^{\prime}$ the $\mathrm{AGG}^{\prime}$ fraction features the same decay kinetics (time constants for the fluorescence and anisotropy decay) as the AGG component. Fitting the data with model 2 yields the thick lines in Fig. 2c, which are in very good agreement with the experiment. Model 2 reproduces in particular the data for the perpendicularly polarised emission in the red spectral region ( $c f$. Fig. 2c, top right). From the fits we obtain that $9 \%$ of the aggregated dimers belong to the $\mathrm{AGG}^{\prime}$ fraction that decays with $\tau_{\text {relax }}=55 \mathrm{ps}$ to the $\mathrm{J}^{\prime}$ state. We want to emphasise that such an improvement of the modelling could not be achieved by incorporating additional exponentials into the AGG anisotropy decay of model 1 . The results of the global analysis of the anisotropic fluorescence decay of (PerAcr) $)_{2}$ according to model 2 are summarized in Table 1, together with the results of a similar analysis for monomeric PerAcr for comparison. Now the value given for $r_{\mathrm{NF}}$ is significant, in contrast to the initial approach. As a matter of fact, only the anisotropy decays of the monomeric PerAcr and the ISO component of (PerAcr $)_{2}$ follow bi-exponentials. Interestingly, for the
Table 1 Results of a global analysis of the decay of the fluorescence anisotropy of $(\mathrm{PerACr})_{2}$ following model 2. For comparison the results from a similar analysis for monomeric PerAcr are listed as well. If not stated otherwise the entries are given with 2 significant digits

\begin{tabular}{llclll}
\hline Oligomer & Conformation & $r_{1}$ & $\theta_{1} / \mathrm{ps}$ & $r_{2}$ & $\theta_{2} / \mathrm{ps}$ \\
\hline PerAcr & - & 0.30 & 460 & 0.09 & 110 \\
& & & & & \\
$(\text { PerAcr })_{2}$ & ISO & 0.13 & 660 & 0.15 & 120 \\
& AGG & 0.082 & 660 & - & - \\
& AGG & $-0.2 \pm 0.1$ & 660 & - & - \\
& NF & $0.4 \pm 0.1$ & - & - & - \\
\hline
\end{tabular}

latter case, the long time constant is identical to those found for the anisotropy decay of the aggregated conformations AGG and $\mathrm{AGG}^{\prime}$.

\section{Discussion}

For the monomeric PerAcr we find a bi-exponential decay of the fluorescence anisotropy. The short component amounts to $110 \mathrm{ps}$ and is ascribed predominantly to the movement of the PBI chromophore relative to the acryl backbone, whereas the long component of 460 ps mainly reflects the rotation of the entire macromolecule including the backbone and the undecylalkyl linker. Similarly, we find a bi-exponential decay of the anisotropy for the ISO conformation of the (PerAcr) $)_{2}$ oligomer. The short time constant of 120 ps is in close agreement with the value of 110 ps found for the monomer, supporting the interpretation given above, whereas the longer time constant amounts to 660 ps which is significantly longer than the 460 ps found for the monomer. This increase reflects the larger moments of inertia of the dimeric form and concomitantly a decrease of the rotation frequencies resulting in a slower decay of the fluorescence anisotropy. This is consistent with the observation that the time constant of $660 \mathrm{ps}$ is reproduced also for the aggregated conformations of (PerAcr) $)_{2}$. Taking a closer look at the initial anisotropies, $r_{1}$, obtained for the aggregated conformations AGG and $\mathrm{AGG}^{\prime}$ reveals a striking difference, i.e. 0.082 vs. -0.2 , between these two species. According to model 2 the $\mathrm{AGG}^{\prime}$ component is associated only with the (sub-) population in the $\mathrm{H}$ state, see Fig. 4, and for this configuration, i.e. (nearly) mutually orthogonal transitiondipole moments for the absorbing $(\mathrm{H})$ and the emitting $\left(\mathrm{J}^{\prime}\right)$ state, one expects an initial anisotropy close to -0.2 in agreement with the observations. However, for optically exciting the aggregated fraction of the (PerAcr) $)_{2}$ dimers it is conceivable that the absorption bands associated with the $\mathrm{J}$ and the $\mathrm{H}$ state are very broad at room temperature. Presumably, excitation of AGG-(PerAcr $)_{2}$ populates both states and the intial value of 0.082 reflects the weighted average of the values of about 0.4 and -0.2 which would be expected only for exciting selectively into the $\mathrm{J}$ or the $\mathrm{H}$ state and subsequent emission from $\mathrm{J}^{\prime}$. This interpretation is in line with results from earlier experiments on a different type of J-aggregate that allowed to excite the $\mathrm{H}$ - and J-bands separately. There, a gradual change of the anisotropy from -0.16 to +0.37 was found upon sweeping the 
excitation wavelength from the $\mathrm{H}$ - to the J-type absorption peak and thereby changing the relative contribution of those states to the total anisotropy. ${ }^{44}$

Finally the value of 0.4 for the initial anisotropy of the NF conformation suggests that in this configuration the transition dipole moments for absorption and emission are oriented parallel with respect to each other. The strong quenching of the fluorescence in the NF conformation might reflect the interaction between the aromatic end groups of the linker with the $\pi$-electron system of the PBI. According to the results from the MD simulations these end groups and the PBI are in close vicinity in the conformation that we have associated with the NF state, and there are several examples in the literature where such an interaction results in an efficient suppression of the emission from the chromophore. ${ }^{40,41}$ However, since the initial anisotropy of $r_{\mathrm{NF}}=0.4$ indicates that the transition-dipole moments of the absorbing and emitting states are oriented parallel with respect to each other, and since in any case the emitting state is associated with $\mathrm{J}^{\prime}$, this suggests that the quenching is more efficient in the higher energy state $\mathrm{H}$.

So far we have developed a picture that connects the experimental results with the structures of the aggregated conformations of (PerAcr $)_{2}$ consistently. However, while the initial anisotropies found for the monomeric PerAcr nicely sum up to 0.39 , i.e. very close to the expected value of 0.4 , the sum of the initial anisotropies found for the ISO-(PerAcr) $)_{2}$ amounts only to 0.28 . This observation is clearly not in accordance with the concept of treating ISO-(PerAcr $)_{2}$ as a pair of two loosely connected, yet independent emitters. In order to address this issue in more detail we compute the anisotropy decay $r(t)$ in the ISO state from our MD simulations. For a given chromophore $r(t)$ can be calculated from the trajectory of its dipole vector $\vec{\mu}(t)$ as ${ }^{45}$

$$
r(t)=0.4\left\langle P_{2}[\vec{\mu}(0) \cdot \vec{\mu}(t)]\right\rangle
$$

where $P_{2}[\cdots]$ is the second Legendre polynomial and $\langle\cdots\rangle$ denotes a phase-space average over different starting points along the trajectory. The result is depicted in the upper curve of Fig. 5. The data can be fitted with a triple exponential function whose amplitudes and decay times are given in the first row of Table 2. Noting that $\theta_{3}=14 \mathrm{ps}$ is shorter than our instrument response time of $30 \mathrm{ps}$, we must assume that the third mode is not visible in our experiments, whereas the decay times of the slow and intermediate component, 1250 ps and 310 ps correspond only roughly to the experimental data (660 ps and $120 \mathrm{ps,}$ see Table 1). In addition the simulation analysis fails to reproduce correctly the experimentally observed amplitudes $r_{1}$ and $r_{2}$. More specifically, this naive interpretation of the MD data cannot reproduce the experimentally observed initial anisotropy $\left(r_{1}+r_{2}\right)=0.28$, see Table 1 .

Yet, a possible mechanism that leads to fast depolarisation of the ISO-(PerAcr) $)_{2}$ signal, and which has not been considered as yet, is homo-energy transfer between the two PBI monomers. A rough estimate based on Förster theory (see ESI $\dagger$ ) yields that the energy transfer between the two PBI chromophores becomes faster than the experimental time resolution if the

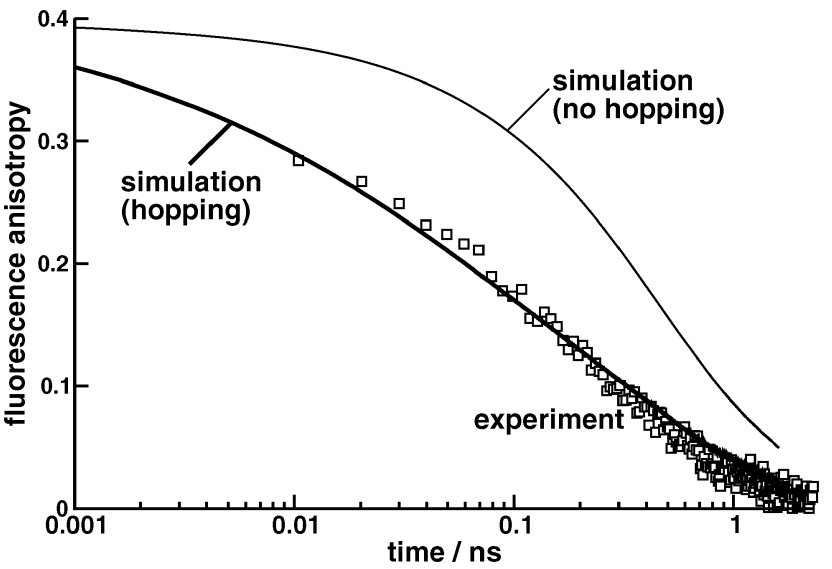

Fig. 5 Anisotropy decay $r(t)$ in the ISO state of (PerAcr $)_{2}$ from experiment (squares, obtained applying eqn (1) to the data from the green spectral range) compared to the simulation analysis with and without hopping of the excitation energy (lower and upper curve, respectively). Good agreement is found only when Förster energy transfer ("hopping") is included in the analysis.

Table 2 Amplitudes and decay times for the triple exponential fits to the anisotropy decay with and without hopping. The corresponding curves are shown in Fig. 5

\begin{tabular}{llrlllr}
\hline & $r_{1}$ & $\theta_{1} / \mathrm{ps}$ & $r_{2}$ & $\theta_{2} / \mathrm{ps}$ & $r_{3}$ & $\theta_{3} / \mathrm{ps}$ \\
\hline No hop & 0.17 & 1250 & 0.2 & 310 & 0.02 & 14 \\
Hop & 0.14 & 804 & 0.13 & 108 & 0.13 & 7 \\
\hline
\end{tabular}

distance between the two pigments decreases below $2 \mathrm{~nm}$, which is predominantly the case for ISO-(PerAcr $)_{2}$, see Fig. $3 \mathrm{~d}$. Hence, it is conceivable that in the ISO conformation fast hopping of the excitation energy between the two PBI chromophores occurs and thereby leads to a fast depolarisation of the signal. We therefore refine the computation of the anisotropy decay by incorporating "excitation hopping" which is an intrinsically quantum-mechanical effect that is not accounted for by our classical MD simulations. In the following we will therefore combine classical trajectory analysis with an appropriate probabilistic hopping model in order to reproduce and interpret the experimental data. For this we track an imaginary excitation which is allowed to hop between the two PBI chromophores during the calculation of the anisotropy decay from the MD trajectory (note that the simulation procedure itself is not modified). The probability for the excitation to remain on the same PBI unit after a short time interval between $t$ and $t+\Delta t$ can be calculated via (see ESI $\dagger$ )

$$
p_{\text {rest }}(t)=\frac{1}{2}+\frac{1}{2} \mathrm{e}^{-2 k(t) \Delta t}
$$

where the hopping rate $k(t)$ depends on the current configuration through ${ }^{35}$

$$
k(t)=\frac{k_{0} \kappa(t)^{2}}{R(t)^{6}} .
$$

Here, $\kappa(t)$ is the orientation factor that takes the mutual alignment of the transition-dipole moments of the two 
chromophores into account, $R(t)$ is the centre-of-mass distance between the two monomers and $k_{0}$ is a physical constant (see ESI $\dagger$ ). We have furthermore taken $\Delta t$ to be small compared to the typical rearrangement time of the molecule such that $k(t)$ can be considered constant between $t$ and $t+\Delta t$. The key aspect of this analysis is that we calculate the hopping rate $k(t)$ instantaneously by using the exact MD coordinates in $\kappa(t)$ and $R(t)$ instead of calculating hopping rates averaged over many molecular conformations as is most commonly done. This allows us to obtain the anisotropy decay with all molecular interactions included and without any further model assumptions. We thus proceed as follows. Without loss of generality, we suppose that the incident radiation is absorbed by chromophore 1. Suppose then that at a later time $t$ the excitation is located on chromophore $a(a=1,2)$. In order to calculate the anisotropy that would be measured if the excitation relaxed at time $t$, we evaluate the analogue of eqn (8) for this situation

$$
r(t)=0.4\left\langle P_{2}\left[\vec{\mu}_{1}(0) \cdot \vec{\mu}_{a}(t)\right]\right\rangle .
$$

Here, $\vec{\mu}_{1}(0)$ denotes the dipole vector of the first chromophore at $t=0$ and $\vec{\mu}_{a}(t)$ is the dipole vector of chromophore $a$ at time $t$. Next we evaluate eqn (9) to obtain the resting probability $p(t)$ in the current configuration where $\Delta t$ is the time step between two consecutive MD frames ( $0.4 \mathrm{ps})$. We generate a random number $x$ between 0 and 1 and if $x>p_{\text {rest }}(t)$, the excitation performs a hop and its position $a$ is changed from 1 to 2 or vice versa. Then the process repeats for the next time frame. The result of this procedure is a triple-exponential decay with amplitudes and parameters as given in the second row of Table 2 and graphically shown in the lower curve of Fig. 5. Again, the shortest decay mode (7 ps) will not be visible in the experiments. The remaining two modes match much closer with the experimental data than the results obtained from the previous analysis without the hopping process. The main difference, however, is that the experimentally invisible 7 ps mode increases strongly in amplitude up to $r_{3}=0.13$. The remaining two modes have almost equal amplitudes, which now match with the experimental observations. Especially, the initial anisotropy of $\left(r_{1}+r_{2}\right)=$ 0.27 almost exactly reproduces the experimental result of 0.28 . Our combination of classical MD simulation with a probabilistic hopping model thus furnishes a strong indication that energy transfer between the PBI units is indeed a relevant process in the non-aggregated conformation of the (PerAcr $)_{2}$ dimers.

\section{Conclusions}

Our study has shown that time-resolved anisotropy in combination with MD simulations and modelling is a powerful tool to study the dynamics in the excited states of extended molecular systems. On the one hand, MD results help to assign molecular configurations to experimental results, on the other hand, those are suitable to motivate and test advanced modelling based on the molecular dynamics simulations. This combined approach allowed us to demonstrate that Förster energy transfer is important in perylene bisimide dimers and - presumably - larger oligomers. We also found that a subtle effect - the breaking of the symmetry of stacked PBI introduced by the connecting chain can have a large impact on the electronically excited states of a macromolecule. We believe that the detection and understanding of effects like the aforementioned ones is a major prerequisite for the design of devices based on PBI derivatives.

\section{Acknowledgements}

M.T. and J.K. thankfully acknowledge financial support by the Deutsche Forschungsgemeinschaft (GRK1640) and the State of Bavaria within the initiative "Solar Technologies go Hybrid". S. G. thanks the Volkswagen Foundation for financial support and gratefully acknowledges computing time granted by the John von Neumann Institute for Computing (NIC) and provided on the supercomputer JUROPA at Jülich Supercomputing Centre (JSC).

\section{References}

1 J. Peet, A. J. Heeger and G. C. Bazan, Acc. Chem. Res., 2009, 42, 1700-1708.

2 J.-L. Brédas, J. E. Norton, J. Cornil and V. Coropceanu, Acc. Chem. Res., 2009, 42, 1691-1699.

3 M. R. Wasielewski, Acc. Chem. Res., 2009, 42, 1910-1921.

4 H. Liu, J. Xu, Y. Li and Y. Li, Acc. Chem. Res., 2010, 43, 1496-1508.

5 T. Weil, T. Vosch, J. Hofkens, K. Peneva and K. Müllen, Angew. Chem., 2010, 49, 9068-9093.

6 J.-L. Bredas and J. R. Durrant, Acc. Chem. Res., 2009, 42, 1689-1690.

7 P. Amsalem, et al., Phys. Rev. B: Condens. Matter Mater. Phys., 2013, 87, 035440.

8 M. Schubert, et al., Adv. Energy Mater., 2012, 2, 369-380.

9 V. Palermo, et al., Adv. Mater., 2010, 22, E81-E88.

10 E. Lang, F. Würthner and J. Köhler, ChemPhysChem, 2005, 6, 935-941.

11 E. Lang, et al., ChemPhysChem, 2007, 8, 1487-1496.

12 J. M. Lim, et al., Chem. Sci., 2013, 4, 388-397.

13 E. Fron, et al., Photochem. Photobiol. Sci., 2008, 7, 597-604.

14 A. Wicklein, A. Lang, M. Muth and M. Thelakkat, J. Am. Chem. Soc., 2009, 131, 14442-14453.

15 X. Zhang, et al., Adv. Mater., 2013, 25, 5791-5797.

16 R. Shivanna, et al., Energy Environ. Sci., 2014, 7, 435-441.

17 Y. Lin, et al., Adv. Mater., 2014, 6, 5137-5142.

18 T. Vosch, et al., J. Phys. Chem. C, 2009, 113, 11773-11782.

19 F. P. Diehl, et al., J. Phys. Chem. Lett., 2013, 5, 262-269.

20 C. C. Hofmann, P. Bauer, S. A. Haque, M. Thelakkat and J. Köhler, J. Chem. Phys., 2009, 131, 144512.

21 C. Hippius, F. Schlosser, M. O. Vysotsky, V. Böhmer and F. Würthner, J. Am. Chem. Soc., 2006, 128, 3870-3871.

22 C. Hippius, et al., J. Phys. Chem. C, 2008, 112, 2476-2486.

23 H. W. Bahng, et al., J. Phys. Chem. B, 2011, 116, 1244-1255.

24 A. Issac, et al., Phys. Chem. Chem. Phys., 2012, 14, 10789-10798.

25 A. Issac, R. Hildner, C. Hippius, F. Würthner and J. Köhler, ACS Nano, 2014, 8, 1708-1717. 
26 F. Schlosser, J. Sung, P. Kim, D. Kim and F. Würthner, Chem. Sci., 2012, 3, 2778-2785.

27 A. Sautter, et al., J. Am. Chem. Soc., 2005, 127, 6719-6729.

28 T. Weil, et al., J. Am. Chem. Soc., 2001, 123, 8101-8108.

29 C. Flors, et al., J. Phys. Chem. C, 2007, 111, 4861-4870.

30 C. C. Hofmann, et al., J. Phys. Chem. B, 2010, 114, 9148-9156.

31 C. C. Hofmann, et al., Phys. Chem. Chem. Phys., 2010, 12, 14485-14491.

32 F. Spreitler, M. Sommer, M. Thelakkat and J. Köhler, Phys. Chem. Chem. Phys., 2012, 14, 7971-7980.

33 B. Hess, C. Kutzner, D. van der Spoel and E. Lindahl, J. Chem. Theory Comput., 2008, 4, 435-447.

34 A. K. Malde, et al., J. Chem. Theory Comput., 2011, 7, 4026-4037.

35 J. R. Lakowicz, Principles of Fluorescence Spectroscopy, Springer, 3rd edn, 2006.
36 V. Marcon, J. Kirkpatrick, W. Pisula and D. Andrienko, Phys. Status Solidi, 2008, 245, 820-824.

37 J. Idé, et al., J. Phys. Chem. B, 2011, 115, 5593-5603.

38 R. F. Fink, et al., J. Am. Chem. Soc., 2008, 130, 12858-12859.

39 V. Marcon, et al., J. Am. Chem. Soc., 2009, 131, 11426-11432.

40 S. Doose, H. Neuweiler and M. Sauer, ChemPhysChem, 2009, 10, 1389-1398.

41 E. L. Rachofsky, R. Osman and J. B. A. Ross, Biochemistry, 2001, 40, 946-956.

42 A. Schubert, et al., J. Phys. Chem. Lett., 2013, 4, 792-796.

43 A. Schubert, et al., J. Phys. Chem. A, 2014, 118, 1403-1412.

44 I. G. Scheblykin, M. A. Drobizhev, O. P. Varnavsky, M. Van der Auweraer and A. G. Vitukhnovsky, Chem. Phys. Lett., 1996, 261, 181-190.

45 H.-S. Tan, I. R. Piletic and M. D. Fayer, J. Chem. Phys., 2005, 122, 174501. 